ANderson, E. S. \& Feux, A. (1953). J. gen. Microbiol. 9, 65-88.

\title{
The Vi Type-determining Phages carried by Salmonella typhi
}

\author{
By E. S. ANDERSON aNd A. FELIX \\ Central Enteric Reference Laboratory and Bureau, Public Health Laboratory Service \\ (Medical Research Council), London, N.W. 9
}

SUMMARY: The properties of the latent phages present in twelve of the thirty recognized Vi-phage types of Salmonella typhi are described. The isolation and characterization of these phages presented great technical difficulties which have been partly overcome.

Nine of the latent phages were shown to control Vi-phage type specificity in the lysogenic strains that carry them. The remaining three phages have not so far been demonstrated to have type-transforming properties.

The latent phages are $O$ phages, as indicated by their lytic action on the Vinegative Sal. typhi strain $\mathbf{O} 901$ and on Sal. gallinarum. By cross-neutralization tests the twelve phages are subdivided into four serological groups, one of which shows a considerable degree of antigenic community with an anti-O phage known to attack many salmonella species belonging to different $O$ groups. No serological relationship was found between any of the latent phages and Vi-phage II.

The Vi-phage type of lysogenic strains of Sal. typhi is an expression of a resistance pattern dependent on the carried phages. These phages seem to confer resistance against unadapted Vi-phage II and simultaneously to induce phenotypic modifications in it to yield specifically adapted Vi-phage preparations.

Artificially prepared Vi-phage types of the typhoid bacillus, produced by the treatment of susceptible strains with type-determining phages, appear to be as stable as the corresponding Vi-types found in naturally occurring lysogenic strains. The fact that world-wide experience with the Vi-phage typing technique over many years has proved its epidemiological reliability suggests that the chances of the typhoid bacillus encountering type-changing phages under natural conditions are remote.

It is shown that the recently proposed method of typing bacteria by identifying the phages they carry is unsuitable for application to Sal. typhi.

The discovery by Craigie \& Yen (1938) of a Vi phage of Salmonella typhi capable of specific adaptation to different strains of the organism placed in our hands a tool for the recognition of Vi-types of the typhoid bacillus that has proved, beyond any doubt, its worth in the investigation of the epidemiological problems associated with typhoid fever. It raised simultaneously questions concerning the nature of the difference between the various Vi-phage types of Sal.typhi, and the mechanism of adaptation of Vi-phage II to these different types of organism.

Craigie (1942, 1946) observed that many strains of $S a l$.typhi carried phages which he called 'latent' or ' $\gamma$ ' phages; he concluded that carriage of these phages made the host organisms untypable with Vi-phage II, and called such strains 'imperfect' Vi forms. Craigie did not make an extensive study of these latent phages. However, he was able to transform Type A into Type D1 with a phage isolated from Type D1. Felix \& Anderson (1951 $a$ ) and Anderson 
(1951) demonstrated that many of the Vi-types of Sal. typhi were lysogenic, and that the phages they carried determined the Vi-type specificity of the strains. The action of these phages on a number of strains of Sal. typhi resulted in a secondary growth of lysogenic organisms of changed Vi-type. Jude, Nicolle \& Ducrest (1951) showed that the phage carried by Type D6 could change Type D1 into Type D6.

Lysogenic cultures are particularly common among strains of Sal. paratyphi $B$ (Nicolle, Grabar \& Gibert, 1946), and Scholtens (1950) made an attempt to 'type' different strains of this organism by identifying the phages they carry. Boyd $(1950,1952)$ suggested this method of phage-typing for Sal. typhi-murium and other salmonella species. Nicolle, Hamon \& Edlinger (1951) showed that type specificity in certain of the phage types of Sal. paratyph $B$, identified according to the typing scheme suggested by Felix \& Callow $(1943,1951)$, depended on lysogenicity, and Hamon \& Nicolle (1951) carried out phage-type transformations of $S a l$. paratyphi $B$ by treating organisms with the phages carried by heterologous types. Felix \& Callow (1951) found that every strain of Sal. paratyphi B could be shown to be lysogenic and briefly described the 'natural' phages characteristic of each of the ten recognized phage types of the paratyphoid-B bacillus. Williams Smith (1951 $a-c)$ published valuable observations on lysogenic strains in five different salmonella species.

The present paper is a detailed description of the phages isolated from various Vi-types of the typhoid bacillus.

\section{EXPERIMENTAL}

\section{Media}

'Bacto' dehydrated nutrient broth (Difco) was used as the standard nutrient basis for all media, which were prepared according to the formula of Craigie \& Felix (1947) and are referred to hereafter as 'Difco' broth and 'Difco' agar; the latter contained $1.3 \%$ New Zealand powdered agar.

\section{Isolation of phages}

During the early phases of the work the organism under test for lysogenicity was seeded lightly into $3 \mathrm{ml}$. of Difco broth and was incubated at $38.5^{\circ}$ for periods varying from 8 to $16 \mathrm{hr}$. The culture was then centrifuged at 3000 r.p.m. for $10 \mathrm{~min}$. and the supernatant was divided into two parts, one of which was heated at $57^{\circ}$ for 40 min. while the other remained unheated. The heated and unheated supernatants were then spotted undiluted on to a lawn of an indicator strain on a Difco agar plate which was incubated at $38.5^{\circ}$ overnight and inspected the following morning for plaques.

Higher yields of phage were obtained by growing the culture under test for lysogenicity together with the indicator strain: $100 \times 10^{6}$ organisms each of the test culture and the indicator strain were added to $20 \mathrm{ml}$. of pre-warmed Difco broth in a 1 oz. screw-capped bottle. The culture was incubated at $38 \cdot 5^{\circ}$ for $8 \mathrm{hr}$. and tested for phage in the way described above, with and without 
heating. The indicator strain employed in all primary tests was the original strain of Vi-phage Type A of Sal. typhi (Craigie \& Yen, 1938). This strain is characterized by its susceptibility to all the Vi typing-phages and has not yet been shown to carry a phage. It was assumed, therefore, that it would probably be susceptible to most, if not all, of the phages carried by the various specific Vi-types. This assumption was fully justified, and Type $A$ has remained the standard indicator strain in these experiments, although it has since been found that several other Vi-types, some of which are themselves lysogenic, are sensitive to many of the latent phages. However, in no instance has it been possible to show that a lysogenic type is susceptible to the phage it carries.

In each case in which a phage has been isolated from a Vi-type by growth of that type with Type A, it has also been observed that the same phage could be obtained, though in much lower titre, from the culture under test grown without the indicator strain.

Table 1 shows the twelve Vi-types of Sal. typhi from which latent phages have so far been isolated by the methods described, together with the designation of the phages, which is an amplification of that suggested in a former paper (Felix \& Anderson, 1951 a). Although latent phages have not yet been isolated from the remaining eighteen recognized Vi-phage types and subtypes of the typhoid bacillus, it is not unlikely that some of them carry typedetermining latent phages. Most of the phages described in this paper form extremely small plaques, some no more than a few microns in diameter. They are readsorbed by the lysogenic organisms emerging as secondary growth from lysates and are sensitive to hitherto unidentified differences in solid media that may inhibit plaque formation altogether. It is possible that the latent phages not yet isolated do not show visible lysis on Type $A$ or on any other of the known Vi-type strains of Sal. typhi, and that they have escaped detection because a suitable indicator strain has not yet been employed.

Table 1. Vi-phage types of Salmonella typhi known to be lysogenic

$\begin{array}{cccc}\text { Vi-phage types } & \begin{array}{c}\text { Designation of } \\ \text { latent phages }\end{array} & \begin{array}{c}\text { Di-phage types } \\ \text { Designation of } \\ \text { latent phages }\end{array} \\ \text { B3 } & b 3 & \mathbf{T} & t \\ \text { D1 } & d 1 & 25 & 2^{\prime} \\ \text { D4 } & d 4 & 26 & \mathbf{2 6}^{\prime} \\ \text { D6 } & d 6 & 28 & 2^{\prime} \\ \text { F2 } & f 2 & 29 & 29^{\prime} \\ \text { K } & k & 30^{*} & 30^{\prime}\end{array}$

* Type 30 was recently identified by one of us (E. S. A.) but has not yet been published: its designation is provisional.

\section{Preparation of high-titre phages}

One of the most difficult problems encountered in this work was that of obtaining the latent phages in sufficiently high titre to be usable for experimental purposes, particularly as antigens to produce antisera in rabbits. While some of the phages, such as $d 1$ and $d 4$, could be isolated with moderate ease, none was easy to grow in titres exceeding $10^{8}$ particles $/ \mathrm{ml}$., and indeed 
only two of the phages, namely $d 1$ and $k$, have ever attained in our hands a titre exceeding $5 \times 10^{8}$. This difficulty was probably caused, at least in part, by the rapid production of lysogenicity in the host organisms as soon as moderately high phage titres were reached; these lysogenic organisms adsorb free phage and the titre of the latter drops. It was found that there was an optimal phage/organism ratio for each phage/indicator-strain system that would produce the maximum titre with the minimum of secondary growth of lysogenic organisms. When this ratio could be determined it was usually possible to obtain good yields of phage by adjusting the initial inocula of phage and organism accordingly. However, the ratio seemed to vary with the volume of the broth medium and the size of the container, and it was only possible to take advantage of this method to a limited extent.

A useful technique was evolved whereby the phages were allowed to establish their own optimal ratios with the host cells during series of subcultures in $20 \mathrm{ml}$. volumes of Difco broth in $25 \mathrm{ml}$. screw-capped bottles. This method was specially useful with the more difficult phages such as phages $d 6, f 2$ and $29^{\prime}$. As a general rule the inoculum into the first bottle consisted of $200 \times 10^{6}$ organisms of Type A and $1 \mathrm{ml}$. of as high-titre a preparation of the phage to be grown as was available. The actual number of particles introduced usually lay between $10^{6}$ and $10^{8}$. The bottle was incubated at $38.5^{\circ}$ for $8 \mathrm{hr}$., its opacity being compared hourly with that of a control bottle inoculated with $200 \times 10^{6}$ organisms of Type A but no phage. At the end of $8 \mathrm{hr}$. the bottles were transferred to the refrigerator where they remained overnight. The following morning $\mathbf{0} \cdot 1 \mathrm{ml}$. was transferred from each bottle, including the control, to $20 \mathrm{ml}$. of fresh pre-warmed Difco broth which was then incubated at $38.5^{\circ}$ for $8 \mathrm{hr}$., the opacity of phage-containing bottles being compared hourly with that of controls as before. At the end of $8 \mathrm{hr}$. the bottles were transferred to the refrigerator. These manipulations were repeated daily, and $1 \mathrm{ml}$. was removed from each experimental bottle at the end of its $8 \mathrm{hr}$. incubation period to be titrated for phage content. All bottles were stored at $4^{\circ}$ when their incubation was completed. With the majority of the latent phages of $S a l$. typhi the phage/organism ratio seemed to adjust itself to an optimum after a few serial transfers, so that massive lysis of the organism occurred to give a maximum yield of phage. Continuation of the transfers beyond this point resulted in a rapid increase in opacity with a drop in phage titre: this stage marked the establishment of lysogenesis in the indicator strain, and tests with the standard Vi-typing phages revealed that the Vi-phage type of the strain had been changed from Type $A$ to a type which depended on the latent phage used. The number of serial transfers required to reach the optimal phage/organism ratio varied with the different phages. Thus, with phage $d 1$, one or two transfers sufficed; with phage $t$, fifteen or sixteen were necessary.

When a culture in a transfer series showed heavy lysis, the culture preceding it in the series was subcultured to bottles containing $150 \mathrm{ml}$. of fresh Difco broth at $38.5^{\circ}$. The amount transferred to each $150 \mathrm{ml}$. was $0.75 \mathrm{ml}$. and a control culture of the organism alone was set up in the same way. The large volumes were incubated at $38.5^{\circ}$ for as long as lysis continued. When it 
seemed that secondary growth of organisms was commencing, incubation was arrested and the preparations were sterilized by the methods listed in Table 2.

Table 2. Methods used for eliminating the propagating strain from preparations of the latent phages of Salmonella typhi

\begin{tabular}{|c|c|c|}
\hline $\begin{array}{l}\text { Heating at } 57^{\circ} \\
\text { for } 40 \mathrm{~min} .\end{array}$ & $\begin{array}{l}\text { Treatment with } \\
0.16 \% \text { toluene } \\
\text { at } 87^{\circ} \text { for } 1 \mathrm{hr} .\end{array}$ & $\begin{array}{l}\text { Filtration through } \\
\text { Gradocol membranes } \\
\text { (750 m } \mu \text {. A.P.D.) }\end{array}$ \\
\hline$d 1$ & $d 6$ & $\mathbf{b 3}$ \\
\hline$d 4$ & $f^{2}$ & $t$ \\
\hline$\cdot$ & $k$ & $\mathbf{2 8}^{\prime}$ \\
\hline - & $\mathbf{2 5}^{\prime}$ & • \\
\hline - & $\mathbf{2 6}^{\prime}$ & . \\
\hline - & $\mathbf{2 9} \mathbf{9}^{\prime}$ & . \\
\hline . & $30^{\prime}$ & . \\
\hline
\end{tabular}

Many of the latent phages were difficult to obtain free from the organisms on which they had been grown. Phages $d 1$ and $d 4$ presented no trouble, as they were resistant to heat. However, all the remaining phages were heatlabile, and only a small proportion of particles survived heating at $57^{\circ}$ for $\mathbf{3 0}$ min. Filtration through a Seitz EK or equivalent pad resulted in losses of up to $95 \%$ or more of these phages; in addition, filtration of the large volumes needed was unreliable as the living bacilli penetrated the pads. Porcelain filters were not used.

The heat-labile phages are all sensitive to ether and chloroform, but some were found to be largely or completely resistant to $0 \cdot 16 \%$ toluene, a concentration which is lethal to $S a l$. typhi when acting for $1 \mathrm{hr}$. at $37^{\circ}$; the excess of toluene was then removed by evaporation under reduced pressure for $1 \frac{1}{2} \mathrm{hr}$. at $37^{\circ}$. Three of the phages were filtered through Gradocol membranes of $750 \mathrm{~m} \mu$. A.P.D. Table 2 shows the methods adopted for eliminating the propagating strain of Sal. typhi from the various phage preparations. Many of the latent phages used in the following experiments were maintained for periods of over 2 years and subjected to repeated single-plaque isolation.

\section{Stability of the latent phages on storage at $4^{\circ}$}

Phages $d 6, f 2,2^{\prime}, 26^{\prime}, 29^{\prime}$ and $30^{\prime}$ were found to be relatively stable on storage for 6 months at $4^{\circ}$. Phages $b 3$ and $28^{\prime}$, which are sensitive to toluene and to heat, are stable at $4^{\circ}$. Phage $k$ is sensitive to heat but not to toluene; it keeps well at $4^{\circ}$. Phage $t$ is not only sensitive to toluene and to heat but also shows slow deterioration in the cold. Of all the phages described in this paper it is the most difficult to prepare and store.

\section{Characteristics of plaques of the latent phages}

Table 3 indicates the average size and characteristics of plaques produced by the latent phages. Drops of a suitable dilution of the phage were spotted on to a lawn of Type A of Sal. typhi on Difco agar plates. The plates were incubated for $7 \mathrm{hr}$. and read with a $\times 10$ aplanat hand-lens in strong indirect 
lighting. The size of the smallest plaques was estimated on the basis that the limit of resolution of a $\times 10$ lens in good lighting conditions is $10 \mu$. The estimation of their size is thus approximate.

\begin{tabular}{|c|c|c|}
\hline Phage & $\begin{array}{l}\text { Average plaque } \\
\text { size (mm.) }\end{array}$ & Plaque characters \\
\hline b3 & $0.05-0.2$ & $\begin{array}{l}\text { Circular with rapid } \\
\text { secondary growth }\end{array}$ \\
\hline$d 1$ & $0 \cdot 1-0 \cdot 2$ & $\begin{array}{l}\text { Circular, rapid central } \\
\text { secondary growth }\end{array}$ \\
\hline$d 4$ & $0 \cdot 1-0 \cdot 2$ & $\begin{array}{l}\text { Circular, rapid central } \\
\text { secondary growth }\end{array}$ \\
\hline$d 6$ & $0 \cdot 01-0 \cdot 1$ & Punctate \\
\hline$f 2$ & $0.01-0.1$ & Punctate \\
\hline$k$ & $0.01-0.1$ & Punctate \\
\hline$t$ & $0.01-0.1$ & Punctate \\
\hline $\mathbf{2 5}^{\prime}$ & 0.2 & $\begin{array}{l}\text { Circular, shallow, } \\
\text { obscured after } 24 \mathrm{hr} \text {. } \\
\text { incubation }\end{array}$ \\
\hline $\mathbf{2 6}^{\prime}$ & $0 \cdot 05-0 \cdot 15$ & $\begin{array}{l}\text { Circular, shallow, often } \\
\text { obscured after } 24 \mathrm{hr} \text {. } \\
\text { incubation }\end{array}$ \\
\hline $\mathbf{2 8 ^ { \prime }}$ & $0 \cdot 05-0 \cdot 2$ & $\begin{array}{l}\text { Circular with rapid } \\
\text { secondary growth }\end{array}$ \\
\hline $29^{\prime}$ & $0 \cdot 01-0 \cdot 1$ & Punctate. \\
\hline $30^{\prime}$ & $0.01-0.1$ & Punctate \\
\hline
\end{tabular}

Phages $d 6, f 2, t, 25^{\prime}, 26^{\prime}, 29^{\prime}$ and $30^{\prime}$ showed inexplicable variation in lysis on solid media, although they regularly produced lysis in broth cultures. Sometimes they would produce no plaques at all on the standard Difco agar; on other occasions all plaques would be in the neighbourhood of $10 \mu$. in diameter; they were rarely visible to the naked eye. This variation in lytic efficiency was apparent, not only on different batches of Difco agar, but even on occasion on different plates of the same batch.

Prolonged efforts were made to eliminate this inconstancy of lysis and to increase plaque size. Full details of these attempts will not be given as they were uniformly negative. However, as a matter of interest it may be mentioned that among the methods tried were the following: addition of various cations $\left(\mathrm{Ca}^{++}, \mathrm{Mg}^{++}, \mathrm{Mn}^{++}\right.$and $\left.\mathrm{Co}^{++}\right)$in a wide range of concentrations; reinforcement of the medium with a range of peptones; addition of glycerol; varying times and temperatures of incubation; varying concentrations of agar base; variations in $\mathrm{pH}$ value; and many others. The agar-layer method of phage titration, first introduced by Gratia (1936) and widely used of recent years in work on the $\mathbf{T}$ group of phages (see Adams, 1950), yielded plaques easily visible to the naked eye even with the phages giving the smallest plaques by our routine method of titration. Unfortunately, the low agar concentration employed for the superficial layer in the Gratia technique invariably resulted in the exudation of a considerable amount of heavily infected liquid which made it difficult and dangerous to handle large numbers of plates. Minute increases 
in the percentage of agar in the superficial layer restored plaques to their original microscopic sizes, and experiments showed that it was not possible to obtain plaques of substantially increased size in the absence of free infected liquid. It was therefore decided that the method was too unsafe for routine use in work with Sal. typhi.

It was found that plaques were larger when the broth culture used for preparing the bacterial lawn was diluted from the usual 1-1.5 $\times 10^{9}$ organisms $/ \mathrm{ml}$. to $0.3-0.5 \times 10^{2} / \mathrm{ml}$. However, as the concentration of organisms in the lawn dropped the efficiency of plating of the phages fell with it, so that the advantage of the gain in plaque size was offset by the fall in the plaque count; this led to the abandonment of the use of diluted cultures for plaque-counting experiments. It was finally observed that pouring Difco agar plates at $100^{\circ}$ provided a medium giving the most uniformly visible and countable plaques with the phages of the micro-plaque group. Pl. 1 illustrates the plaque sizes of these phages compared with those of Vi-phage $A$.

Titration of the phages was carried out by spotting the various dilutions with a loop on to a bacterial lawn of the indicator strain. Six spots of each dilution were applied. A standard loop was employed with an internal diameter of $3 \mathrm{~mm}$. which delivered in the hands of an experienced technician a drop of great uniformity with a volume very close to $0.01 \mathrm{ml}$. spreading to a diameter of about $1.0 \mathrm{~cm}$. on the bacterial lawn. Counts were first carried out after incubation at $38.5^{\circ}$ for $7 \mathrm{hr}$. and were checked after a further $8 \mathrm{hr}$. incubation. Each area covered by the drop, as seen through the bottom of the covered Petri dish, was divided into squares with red ink, and counts were made with a $\times 10$ hand-lens in strong oblique lighting. An electric counter tripped by a foot-switch was employed.

\section{Lytic spectrum of the latent phages}

Table 4 shows the lytic spectrum of the latent phages on the stock strains of the recognized Vi-types and subtypes of Sal. typhi and on two Vi-negative control strains, namely, Sal. typhi 0901 and Sal. gallinarum. The two Viphage Types $\mathrm{D} 5$ and $\mathrm{H}$, both rare types, were omitted from Table 4, as the available cultures of these type strains were in an advanced state of 'degradation' at the time the experiments were carried out. Table 4 demonstrates that many Vi-types, some of which are themselves lysogenic, are sensitive to the latent phages. The phages have been divided into groups according to their serological reactions which will be discussed later. The corresponding twelve Vi-types carrying them are, for convenience of presentation, grouped similarly.

A number of conclusions can be drawn from the lytic reactions shown in Table 4. In the first place it is evident that several different Vi-types carry phages that are related serologically, in lytic spectrum, and, as will be shown later, in a number of other properties. This applies particularly to phages $b 3$ and $28^{\prime}$, phages $d 1$ and $d 4$, and phages $d 6, f 2,29^{\prime}$ and $30^{\prime}$. If the characterization of latent phages were used as the method of strain identification, as suggested by Scholtens (1950), Boyd (1950) and Boyd, Parker \& Mair (1951), the eight clearly defined Vi-types carrying these phages would be reduced to 


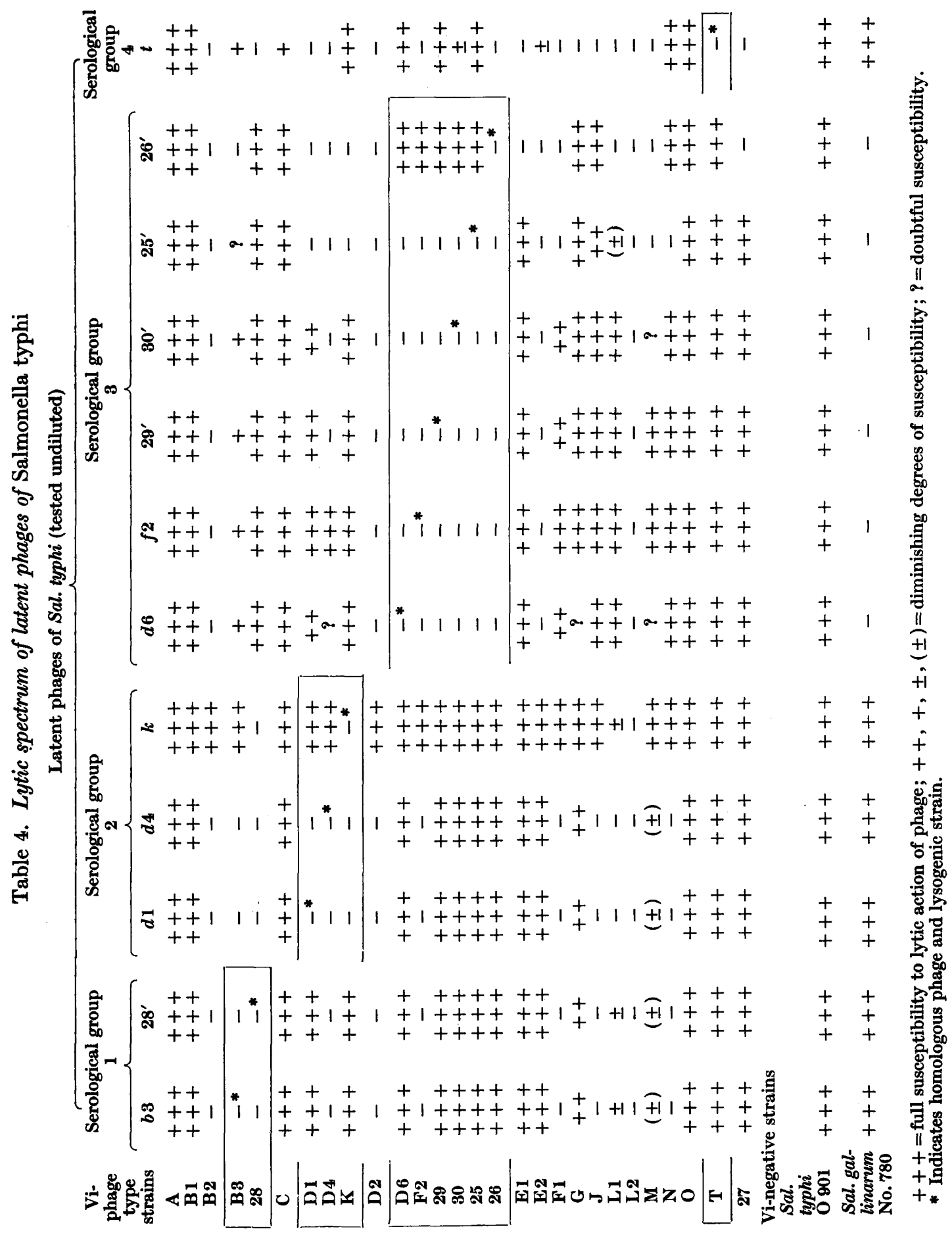


three types only, one in each group, as all strains carrying one of a group of closely related phages would appear to be identical. The importance of this will be made clear in the Discussion.

It is also apparent from Table 4 that the latent phages in any one group show closely similar lytic spectra. Exceptions to this rule are, phage $k$ in group 2 and phages $25^{\prime}$ and $26^{\prime}$ in group 3 . However, as will be shown later, these three phages exhibit weaker group serological relationships than do the other members of the respective groups.

Table 4 also shows that these phages can attack Vi-negative strains of Sal. typhi. Strain O901, a Vi-negative variant used by one of us (A. F.) for a number of years as a control culture to detect the presence of contaminant $O$ phages in Vi-phage preparations, is susceptible to all of the latent phages. That some of these phages can also attack salmonella types other than Sal. typhi is illustrated by the sensitivity of Sal. gallinarum No. 780 to phages $b 3,28^{\prime}, d 1, d 4$, $k$ and $t$.

\section{Preparation of lysogenic strains}

Two methods were used for the preparation of lysogenic strains. The first consisted in subcultivating the secondary growth from the phage-inoculated area resulting from the application of a drop of undiluted phage to a lawn of the indicator strain. This procedure was successful when the phage titre was sufficiently high for the lysate to yield confluent lysis; with phages producing plaques as small as those of phages $d 6, f 2,29^{\prime}$ and $30^{\prime}$ this required a titre of $1 \times 10^{7}$ or higher. For this reason the method was most successful with phages $d 1, d 4, k, 25^{\prime}$ and $26^{\prime}$, all of which attained or exceeded this titre or whose plaques were large enough to cause confluent lysis in lower titres. The secondary growth was well developed after plates had been incubated for $16 \mathrm{hr}$. at $38 \cdot 5^{\circ}$. A sweep from the centre of the phage-inoculated area was transferred to $2 \mathrm{ml}$. of Difco broth which was incubated at $38.5^{\circ}$ until it attained an opacity of 1-1.5 $\times 106$ organisms. It was then tested with the routine typing phages to identify type changes and simultaneously subcultured to a Dorset egg slope for storage.

The second method was used for the phages that commonly gave titres below $1 \times 10^{7}$ and was essentially similar to that described earlier for the production of high-titre lysates by the transfer of $0.1 \mathrm{ml}$. inocula through a series of $20 \mathrm{ml}$. Difco broth bottles. The first bottle contained $20 \mathrm{ml}$. of Difco broth to which was added $1 \mathrm{ml}$. of the most concentrated lysate available of the phage to be used and $200 \times 10^{6}$ organisms in the logarithmic phase of the strain it was wished to make lysogenic. The rapidity of emergence of the lysogenic organisms varied with the phages used in exactly the same way as did the speed of production of high titre lysates, but the sequence was always the same: a phase without detectable lysis was succeeded by one of heavy lysis which gave way to a third phase of normal growth of the bacteria. The second phase marked the period of maximum destruction of susceptible organisms by the phage and the establishment of lysogenicity in the survivors; the increase of turbidity in the third phase was due to the multiplication of lysogenic organisms resistant to phage action. Each broth culture in the 
series was subcultured to a Dorset egg slope at the end of its $8 \mathrm{hr}$. incubation. After overnight incubation at $38.5^{\circ}$ these slopes were tested for Vi-phage type and stored thereafter at room temperature in the dark. These subcultures reflected the successive stages in the transition of the whole culture from phage sensitivity to lysogenicity. Single-colony subcultures were also prepared of strains on which lysogenicity had been impressed, and these were tested after varying periods of storage to determine the stability of the artificially induced lysogenic state.

A simple technique was later evolved which often resulted in the production of lysogenic strains in a single manipulation, yet did not require initial phage titres higher than $10^{6} / \mathrm{ml}$. The procedure was as follows: a very small number of bacterial cells, between 1 and 10, was introduced into $1 \mathrm{ml}$. of a lysate containing between $10^{8}$ and $10^{7}$ phage particles $/ \mathrm{ml}$. The culture was then incubated at $38 \cdot 5^{\circ}$ for about $24 \mathrm{hr}$. The resulting culture was usually lysogenic and showed the change in Vi-phage type characteristic of the latent phage employed. It seems probable that the following sequence of events occurred: under the initial conditions of the experiment the probability of collision between a phage particle and the organisms was so small that the culture was never sterilized by the phage, even when the experiment was duplicated in a large number of tubes containing an average of 0.5 organism or less each (that is, the chances of each fertile tube containing more than one organism were low). Presumably the bacteria were able to multiply sufficiently to keep ahead of their rate of destruction by phage until the density of the culture reached a point at which a large number of organisms encountered phage particles. Most of the sensitive organisms would then be eliminated in a few cycles of phage multiplication and lysogenicity established in the survivors.

\section{Changes in bacteriophage reactions following impressed lysogenicity}

Table 5 summarizes the changes brought about artificially by treating various Vi-phage types of Sal. typhi with the latent phages of other type strains. Phages $b 3$ and $28^{\prime}$ have not so far been shown to have type-transforming properties detectable by the test phages employed in this investigation. Phage $k$ does not change the Vi-phage type of Type A, but makes it resistant to the polyvalent anti-O phage shown in Table 5 that is in routine use in this laboratory as a control reagent to indicate that cultures under investigation belong to the salmonella group (see Felix \& Callow, 1943). Phages b3, 28' and $k$ have, therefore, not been included in the table.

The range of Vi-type variations outlined in Table 5 by no means exhausts the potentialities of the latent phages of Sal. typhi. A limited selection only of the type strains susceptible to these phages were made lysogenic. Type $A$ was most frequently used because it was the standard indicator strain employed for detecting and propagating the latent phages. It appeared to be susceptible to all the phages carried by the different types of the typhoid bacillus. Types $C$, $\mathbf{E} 1, \mathbf{O}$, and $\mathbf{T}$ have also a wide range of susceptibility to these phages.

The change of Type F1 to F 2 with phage $f 2$ was one of the earliest trans- 
formations carried out, and the work connected with it helped to clarify the enigma of what constituted Vi-type specificity in the lysogenic types of $\mathbf{S a l}$. typhi (see Felix \& Anderson, 1951 $a$; Anderson, 1951).

Table 5. Artificial changes in bacteriophage reactions of Salmonella typhi

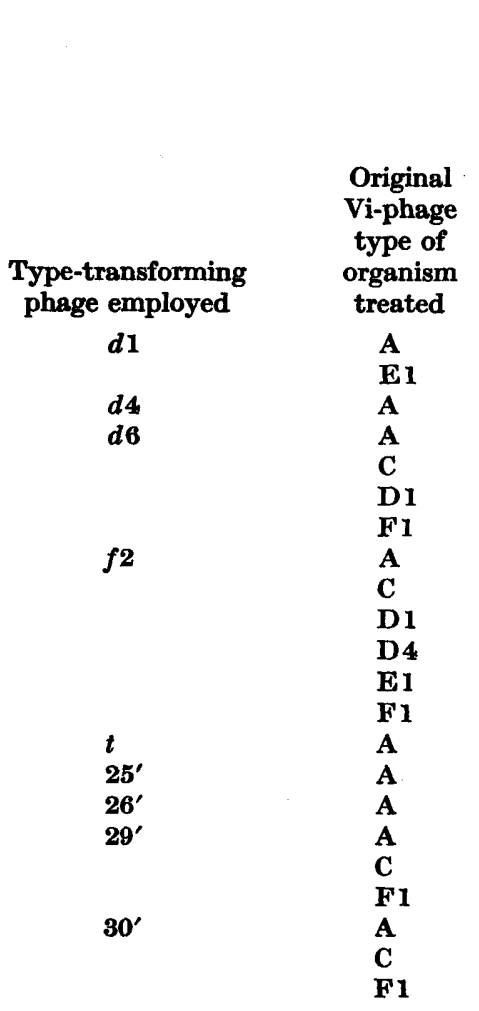
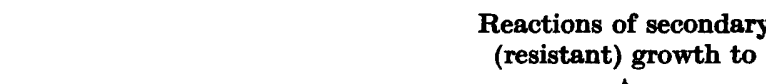

(resistant) growth to

Vi-phages I and IV
of Craigie \& Yen

(1938) (Routine test

dilution
[R.T.D.] $=1:$ 100)

Polyvalent

Vi-phage type of
secondary (resistant)

growth

$\overbrace{I}^{\text {[R.T.D.] }=1: 100)}$

D1

Untypable Vi strain

D1

Untypable Vi strain

D6

F2

$\mathbf{2 9}$

30

D6

Untypable Vi strain

New subtype of $\mathbf{E}$ group

F2

$\mathbf{T}$

25

26

29

30

F2

29

30

F2

salmonella

anti-O phage

$\begin{array}{lll}+ & - & + \\ \pm & - & + \\ + & - & + \\ + & \pm & \pm \\ + & \pm & \pm \\ + & - & \pm \\ + & - & \pm \\ + & \pm & \pm \\ + & \pm & \pm \\ + & - & \pm \\ + & - & \pm \\ \pm & \pm & \pm \\ + & - & \pm \\ + & + & \pm \\ + & - & \pm \\ + & - & \pm \\ + & \pm & \pm \\ + & \pm & \pm \\ + & - & \pm \\ + & + & \pm \\ + & + & \\ + & - & \end{array}$

Controls

$\begin{array}{lll}\begin{array}{c}\text { Reactions to } \\ \text { Vi-phages I } \\ \text { and IV and }\end{array} & \begin{array}{c}\text { Original Vi- } \\ \text { type strains }\end{array} & \text { A } \\ \text { anti-O } & & \text { D1 } \\ \text { phage of } & & \text { D4 } \\ & & \text { E1 } \\ & & \text { F1 } \\ & \text { Naturally oc- } & \text { D6 } \\ & \text { curring Vi- } & \text { F2 } \\ & \text { type strains } & \text { T } \\ & \text { correspond- } & 25 \\ & \text { ing to those } & 26 \\ & \text { produced } & 29 \\ & \text { artificially } & 30\end{array}$

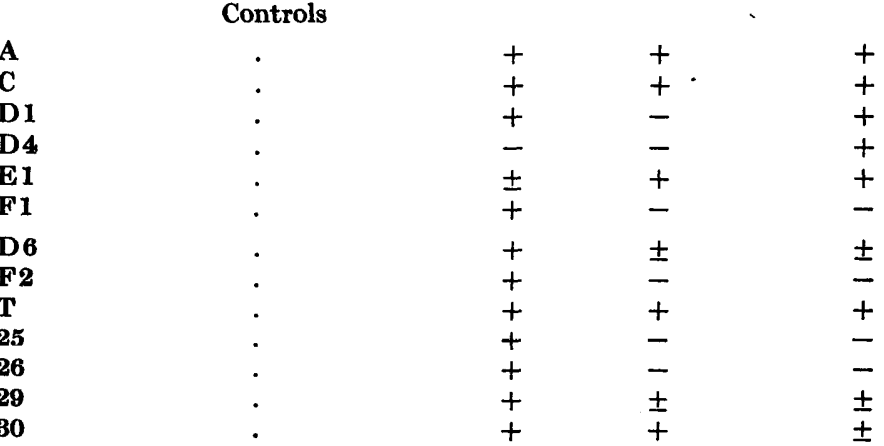

$+=$ sensitive to phage in R.T.D.; $\pm=$ partially resistant to phage in R.T.D.; $-=$ resistant to phage in R.T.D.

There seems to be no reason why a type transformation should not be possible in every phage type of Sal. typhi that is sensitive to the natural phages, although many of the types so produced would not be identifiable 
with the existing set of adapted Vi typing-phages and would be designated as 'untypable Vi-strains' according to the more recent definition of this term (Felix \& Anderson, $1951 b$ ).

The changes in Vi-type are often accompanied by changes in susceptibility to Vi-phages that are serologically unrelated to Vi-phage II and, more strikingly, by changes in sensitivity to the polyvalent anti-O phage referred to above. The significance of these correlated changes in phage sensitivity following the establishment of lysogenesis with a single phage will be discussed later.

It can be seen from Table 5 that as a rule the laboratory made Vi-type was identical with the corresponding naturally occurring type, but certain differences occurred to which attention should be drawn:

(1) Phage $30^{\prime}$ acting on Type A produced Type 29, but this differed from the naturally occurring Type 29 and that prepared from Type A with phage $29^{\prime}$ in being fully sensitive to Vi-phage IV.

(2) Conversely, Type 30 prepared from Type C with phage $29^{\prime}$ differed from the natural Type 30 and that prepared from Type $\mathrm{C}$ with phage $30^{\prime}$ in being partially resistant to Vi-phage IV.

In this case two latent phages, $29^{\prime}$ and $30^{\prime}$, acting on the same strains produce Vi-phage types that are identical except for sensitivity to Vi-phage IV. As will be shown later, the relationship between phages $29^{\prime}$ and $30^{\prime}$ is so close that this slight difference in the phage-sensitivity pattern of the lysogenic resistant growths they produce is, so far, the only means of distinguishing between them.

The stability of artificially prepared Vi-phage types of Salmonella typhi

Hamon \& Nicolle (1951) prepared artificially a number of phage types of Sal. paratyphi $B$ by methods similar to those described here. They demonstrated, however, that the impressed lysogenicity which controlled the phage type was less stable than that occurring naturally. In the present work, a large number of strains of Sal. typhi which were made lysogenic artificially was re-examined after storage for about 18 months or more, and no evidence was found that the laboratory made lysogenicity was any less stable than that occurring naturally. The Vi phage reactions of the cultures remained unchanged, and the phages that had been grafted on to the organisms could be grown from them. The following examples may serve to illustrate this point.

Table 5 shows that Type D1 is converted into Type D6 by phage $f 2$. Type D1 normally carries a heat-stable phage, whereas phage $f 2$ is thermolabile. A phage preparation was grown from a fresh single-colony culture of the artificial Type D6 about 20 months after its transformation, and was divided into two portions, one of which was heated at $60^{\circ}$ for $40 \mathrm{~min}$., while the other was treated with toluene. Both portions were titrated on Type A, single plaques cut and the resulting phages identified. The heated portion contained only phage $d 1$ but the toluene-treated fraction contained both phage $d 1$ and phage $f 2$. The organism had thus been made doubly lysogenic and this double lysogenicity had remained stable for 20 months. The stability 
of the acquired lysogenicity has also been demonstrated by tests of large numbers of single-colony cultures.

On the other hand, it has been pointed out in previous papers (Felix \& Anderson, 1951 $a$; Anderson, 1951) that the state of lysogenicity in naturally occurring strains of Type F2 is unstable, and that Dorset egg cultures of Type F2 stored for long periods in the dark at room temperature throw off phage-free organisms, which belong to Type F1. The same phenomenon was found in cultures of Type F2 prepared artificially from Type F1 with phage $f 2$.

The evidence at present available, therefore, suggests that the state of lysogenicity produced artificially by treating the typhoid bacillus with typedetermining phages is as permanent as that found in naturally occurring lysogenic strains; and indeed, it seems probable that there is no difference between naturally occurring and artificially produced lysogenicity.

\section{SERological REACTIONS}

Preparation of antiphage sera

Antisera were prepared against these phages at first by intraperitoneal and later by intravenous inoculation. Intraperitoneal inoculation was employed because it was feared that lysates containing typhoid antigens might be too toxic when injected intravenously into rabbits. Dosage commenced at $2.5 \mathrm{ml}$. of undiluted lysate, followed by $5.0 \mathrm{ml}$. after a 3-day interval; subsequent doses were 10, 20,30,30 and $30 \mathrm{ml}$. at alternating intervals of 3 and 4 days, to a total dosage of about $130 \mathrm{ml}$. of undiluted lysate. The rabbits were bled 5 days after the last inoculation and again 2 days later.

Intravenous inoculation commenced with $2.0 \mathrm{ml}$. of $1 / 10$ phage dilution, followed by $2.0 \mathrm{ml}$. of $1 / 5,1 / 2.5$ and $1 / 1.25$ dilutions. These doses were followed by $2,3,4,4,5,5$ and $5 \mathrm{ml}$. doses successively of undiluted lysate at alternating 3 and 4 days intervals. The rabbits were bled 5 and 7 days after the last inoculation. No toxic reactions occurred in the rabbits inoculated intravenously.

A preliminary bleeding was carried out on all rabbits before immunization with phages. The resulting sera (I bleedings) were always tested in parallel with the post-inoculation sera (II and III bleedings) when the latter were first titrated. When subsequent tests were carried out with an antiserum of low titre a low dilution of the $I$ bleeding from the same rabbit was always included in the series in order to exclude non-specific neutralization of the phages.

\section{Neutralization tests}

The phages and homologous antisera were mixed, each in $0.5 \mathrm{ml}$. quantities, in appropriate dilutions made up with Difco broth. Controls were included of the corresponding I bleedings and of Difco broth, each mixed with the same amount of phage. The dilution of phage used throughout was that which yielded roughly 50 to 100 plaques after being mixed with an equal volume of Difco broth. The mixtures of phage and antisera were incubated at $38.5^{\circ}$ for $3 \mathrm{hr}$. and then kept in the refrigerator overnight. The following morning they 
were spotted with a standard loop delivering a volume of $0.01 \mathrm{ml}$. on to a bacterial lawn of the indicator strain, and plaque counts were made after 7 and $15 \mathrm{hr}$. incubation. Once an orientating titration of this type had been performed, more precise estimates of the serum titres could be arrived at by more closely spaced titrations between selected dilutions. The titre of an antiphage serum was arbitrarily fixed as being the dilution that neutralized about $90 \%$ of the homologous phage particles under the conditions of the experiment.

As the result of these preliminary titrations it was possible to select suitable dilutions of the antisera for cross-tests with heterologous phages. A first crosstest was set up using only two dilutions of the sera, one equal to their titres with the homologous phages, the other up to ten times as strong as this. The phages were also used in two dilutions in this test, one yielding about 50 to 100 plaques on the indicator strain, the other in ten times this concentration. The orientating cross-tests gave an indication of the serological relationships between the various phages, and further tests were carried out with selected groups of phages using a wider range of serum dilutions. Tables $6 \mathrm{~A}, 6 \mathrm{~B}$ and 7 are examples of cross-neutralization tests showing complete serological identity between some phages and less close relationships between others.

Tables $6 \mathrm{~A}$ and $6 \mathrm{~B}$, which are to be read in conjunction, show that an antiserum against phage $d 1$ or phage $d 4$ is able to neutralize either phage to an equal titre. Overlapping cross-reactions of varying degree occur between phages $d 1$ and $d 4$ on the one hand, and $k$ and anti-O no. 2 on the other. The extent of overlapping appears to be greater in the sera of rabbits immunized by the intraperitoneal route than by the intravenous route. In addition, there are variations in the degree of overlapping in the sera of individual rabbits immunized by either route. Of the four sera shown in Table 6B, those against phages $d 1$ and anti-O no. 2 were prepared by intraperitoneal inoculation and anti- $d 4$ and anti- $k$ by intravenous inoculation.

The experiments summarized in Table 7 represent the best results obtained with a group of phages (group 3) that presented great technical difficulties to which reference has been made earlier. Because of their minute plaque sizes and variability of lysis, frequent failures were experienced in neutralization tests: on a number of occasions no plaques could be seen even in the control areas free from antiserum, although preliminary titrations carried out a day or two earlier had yielded countable plaques. After many disappointments it was finally decided to abandon a strictly quantitative examination of the serological relationships between these phages, and to carry out the cross-tests with undiluted phages or low dilutions of them. Because of the conditions of performing the experiments, therefore, counts could be made only when strong neutralization occurred. Nevertheless, the results were clear-cut, in spite of the fact that the figures indicating overlapping reactions are approximate. Phages $d 6, f 2,29^{\prime}$ and $30^{\prime}$ are serologically identical, and are closely related to phage $25^{\prime}$. Phage $26^{\prime}$ is less closely related to phages $d 6, f 2,29^{\prime}$ and $30^{\prime}$ than is phage $25^{\prime}$; and phages $25^{\prime}$ and $26^{\prime}$ have a close serological affinity. The phages in group 3 were mostly poor antigens giving antisera with titres often 


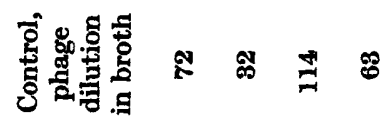

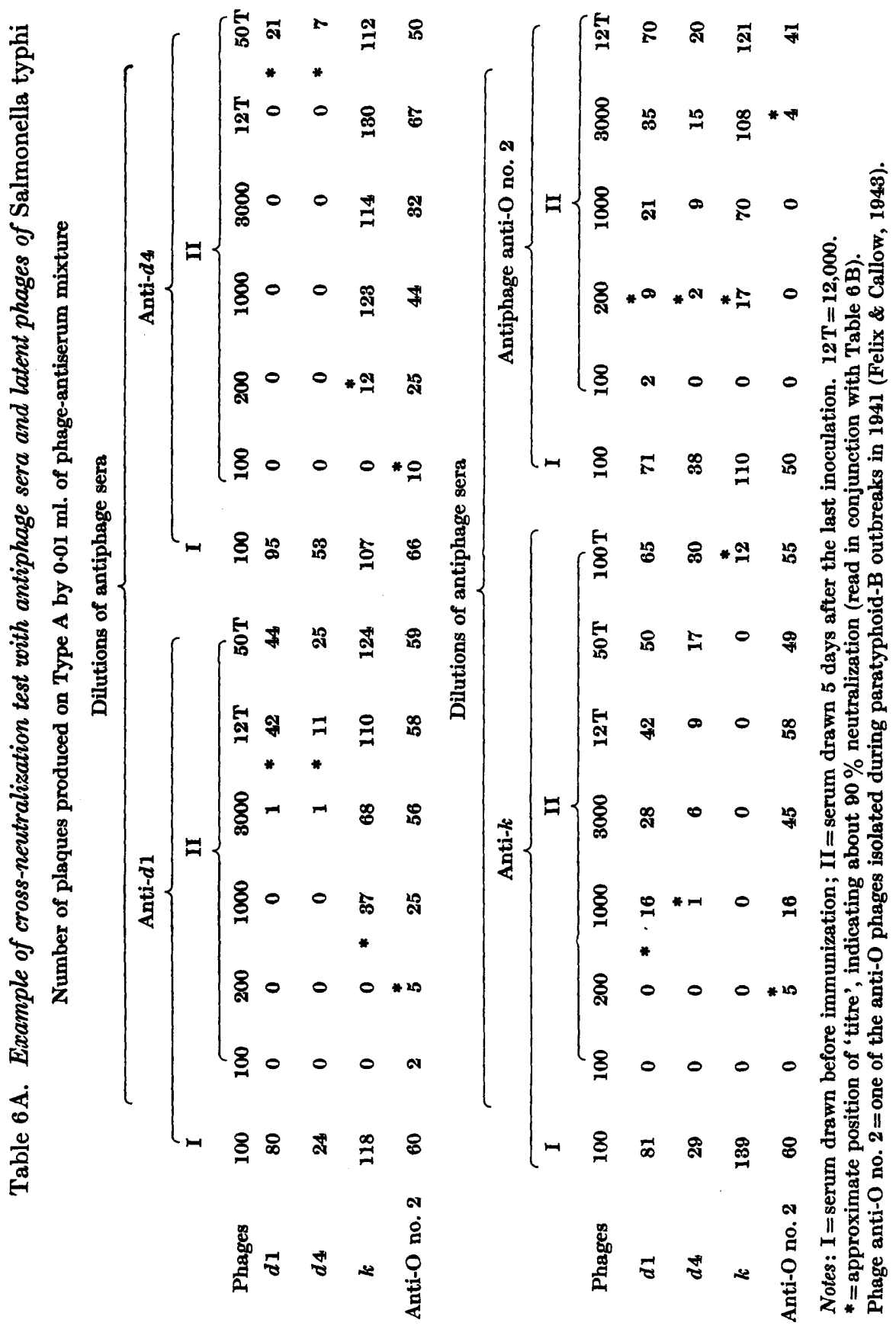


Table 6B. Comparative strength of the four homologous and heterologous neutralizing sera recorded in Table $\mathbf{6 A}$

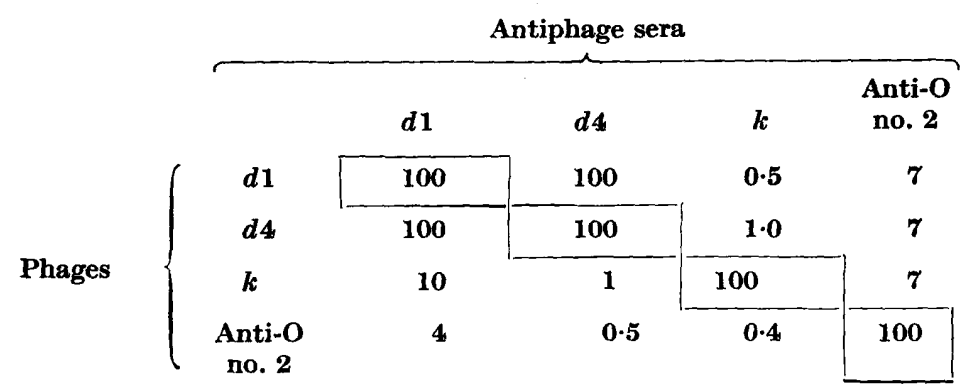

Note: the figure 100 indicates the strength of neutralization of a phage by the homologous antiserum, determined from the 'titre' as defined in the text.

Table 7. Approximate percentage neutralization of the six latent phages of group 3 by homologous and heterologous antisera

\begin{tabular}{|c|c|c|c|c|c|c|c|}
\hline & & & & Antip & era & & \\
\hline & & $d 6$ & $f 2$ & $29^{\prime}$ & $30^{\prime}$ & $\mathbf{2 5}^{\prime}$ & $\mathbf{2 6}^{\prime}$ \\
\hline & $d 6$ & 100 & 100 & 100 & 100 & 90 & 66 \\
\hline & $f 2$ & 100 & 100 & 100 & 100 & 90 & 66 \\
\hline Phages & $29^{\prime}$ & 100 & 100 & 100 & 100 & 20 & 33 \\
\hline & $30^{\prime}$ & 100 & 100 & 100 & 100 & 66 & 33 \\
\hline & $\mathbf{2 5}^{\prime}$ & 50 & 10 & 100 & 20 & 100 & 50 \\
\hline & $\mathbf{2 6}^{\prime}$ & 10 & 10 & 33 & 10 & 100 & 100 \\
\hline
\end{tabular}

as low as $1: 20$, but the cross-neutralization tests were adequately controlled by low dilutions of the corresponding I bleedings.

These tests made it possible to separate the latent phages of Sal. typhi into the serological groups shown in Table 8. In accordance with the analysis of the experiments summarized in Tables $6 \mathrm{~A}, 6 \mathrm{~B}$ and 7 serological groups 2 and 3 were each divided into subgroups $(a)$ and $(b)$. In each case subgroup $(a)$ comprises the phages that are serologically identical, while subgroup (b) contains those less closely related to subgroup $(a)$.

Table 8. Serological groups of latent phages of Salmonella typhi

Group 1

Phage b3 Phage 28'
Group 2

(a) $\left\{\begin{array}{l}\text { Phage } d 1 \\ \text { Phage } d 4\end{array}\right.$

(b) Phage $k$

[Group 2 is related]

to Salmonella phage anti-O no. 2

\section{Group 3}

(a) $\left\{\begin{array}{l}\text { Phage } d 6 \\ \text { Phage } f 2 \\ \text { Phage 29' } \\ \text { Phage 30' }\end{array}\right.$

(b) $\left\{\begin{array}{l}\text { Phage 25' } \\ \text { Phage 26' }\end{array}\right.$
Group 4

Phage $t$ 
The fact that eight of the nine latent phages so far shown to have Vi typedetermining properties belong to two serological groups, one of which contains six of the phages, is of considerable interest. Moreover, these serological relationships are usually associated with similarities in the size and characters of plaques, resistance to heat and chemical agents, efficiency as antigens in rabbits and the Vi type-determining properties of the phages. These points will be referred to again in the Discussion.

\section{DISCUSSION}

The discovery that strains of Sal. typhi could be distinguished from each other by adapted preparations of Vi-phage II (the routine typing phages of Craigie \& Yen, 1938) aroused a considerable amount of conjecture as to what determined the differences between the various Vi-phage types. No demonstrable antigenic differences exist between them (Felix, 1941), nor has it been possible to show differences in virulence (Felix \& Anderson, 1951 b). The first observations of importance in this field were made by Craigie (1942, 1946), who isolated from Type D1 a phage which he called a 'latent phage' or ' $\gamma$ agent'. He reported that this phage transformed Types A and E1 into Type D1.

The significance of Craigie's observations does not seem to have been recognized, and this work was not followed up. The next advance resulted from the demonstration that Type F 1 appeared in old Dorset egg cultures of Type F 2 stored for long periods in the dark at room temperature, and that, in logarithmically growing mixtures of Types F1 and F2, Type F1 disappeared (Anderson, 1951). This phenomenon was found to be caused by a phage which was named phage $f 2$; this phage could lyse Type $F 1$ and could also convert it into Type F2 with the simultaneous production of lysogenicity. In old cultures of Type F 2 some of the cells lost the phage and became Type F 1. These findings and parallel features observed in a number of other Vi-types of the typhoid bacillus were briefly reported in an earlier publication (Felix \& Anderson, 1951 a). Observations similar to our own have been published by Nicolle and his colleagues with the paratyphoid-B Vi-phage types (see Nicolle et al. 1951; Hamon \& Nicolle, 1951), and with Type D1 of Sal. typhi which Jude et al. (1951) were able to change to Type D6 with phage $d 6$.

It is apparent that the determination of Vi-phage type is often dependent on lysogenicity. Moreover, the number of types that can be defined by these phages, which we have designated 'latent' phages in accordance with Craigie (1946), is larger than the number of phages so far isolated. Thus, nine phages have so far been implicated in the type determination of Sal. typhi strains, but some have been shown to be capable of producing several Vi-phage types, the type evolved depending on the original Vi-phage type of the strain used as substrate for the phage. For example, phage $f 2$, acting on Types $A, C, D 1$, D4, E 1 and F1, will produce Types $29,30, D 6$, an untypable Vi strain, a new subtype of the $\mathbf{E}$ group and Type $\mathbf{F} 2$ respectively (see Table 5). The resulting cultures of changed Vi-type become lysogenic and phage $\mathrm{f2}$ can easily be regained from them. As far as can be determined most of the artificially 
prepared types, with a few exceptions some of which have been discussed on p. 76, are identical with those occurring naturally and, up to the present, seem to be quite stable.

\section{Vi-phage type specificity}

The demonstration of these type-changing phages has thrown some light on the nature of the differences in the typhoid bacillus that distinguish the various Vi-phage types and the mechanism of the adaptation of Vi-phage II to the different Vi-phage types. It can be assumed that lysogenicity is responsible for the differences between at least nine of the thirty known phage types, and, as has been pointed out previously (Felix \& Anderson, $1951 a$; Anderson \& Felix, 1952), there can be little doubt that in these types Vi-phage type specificity is an expression of a resistance pattern resulting from the achievement of the state of lysogenicity. It seems probable that the whole range of Vi-typing phages are phenotypic modifications of Vi-phage II (Anderson \& Felix, 1952, 1953). This modification is sufficiently profound to make different adapted preparations, when used in the routine test dilutions, behave in a lytic sense towards different Vi-types of Sal.typhi as if they were distinct phages. However, all Vi-types of the typhoid bacillus will adsorb all the adapted Vi-typing preparations irrespective of the type specificity of the latter, lysis only taking place when the Vi-phage type of the organism and the specificity of the phage coincide (Craigie, 1940). It can therefore be assumed that the resistance to certain Vi-typing preparations that results from the acquisition of lysogenicity and defines the change in Vi-type is caused by a block in the multiplication of heterologous adapted Vi-phage II, and that this block is at some stage in the multiplication of Vi-phage II subsequent to adsorption. As the type-determining phages are $O$ phages and the changes in Vi-type are accompanied also by changes in sensitivity to other Vi-phages than Vi-phage II, and, more significantly, by changes in sensitivity to salmonella anti-O phages, it seems probable that Vi-type specificity is a property that does not reside in the Vi antigen, although the latter is necessary for the initial attachment of Vi-phage II to the organisms. This has been discussed in a previous paper (Anderson \& Felix, 1953).

We have not been able so far to isolate phages from eighteen Vi-types, and the phages carried by three of the lysogenic types (B 3, 28 and K) do not appear to have Vi type-determining properties. Bearing in mind the diffculties encountered in the isolation of a number of the type-determining phages, it is possible that types that have not yet yielded such phages may do so when an effective technique has been evolved for their demonstration. On the other hand, there may also be other, as yet unidentified, processes in the bacterial cell, such as mutation, causing evolution of phage resistance and thus controlling Vi-type specificity.

One can speculate as to the nature of the change in Type $A$ that results in the evolution of a different Vi-type after the production of lysogenicity. If it can be assumed that the focus that determines Vi-type specificity in the bacterial cell and which may be called the 'type-determinant receptor' must 
fit a complementary pattern in the phage before the latter can complete its cycle of multiplication, it must be concluded that this focus has a sufficiently complex pattern in Type $A$ to enable any adapted Vi-typing phage preparation to fit it. The impressing of lysogenicity on Type A with one of the typedetermining phages results in a modification of the pattern, perhaps by obscuring certain groups, so that only one or a few adapted preparations can now fit the pattern sufficiently closely to continue their multiplication. When lysogenicity is lost the occluded groups are re-exposed and the culture becomes Type A once more. This explains at least some of the phenomena previously referred to under the terms 'degradation' and 'degraded Vi strains' (Craigie \& Felix, 1947; Felix \& Anderson, $1951 b$; Anderson \& Felix, 1953). On this hypothesis it would be expected that the change from a lysogenic specific type to Type A would occur in a single step coinciding with the loss of the typedetermining phage. An observation on a strain of Type D6, isolated in November 1951, from a case of typhoid fever, has recently shown that this is so: a subculture of this strain stored at room temperature in the dark for about 12 months was found to have thrown off phage-free variants giving the reactions of Type A. These variants were easily re-converted into Type D6 by the phage carried by the parent strain.

It cannot be inferred that the receptor defining Vi-type in phage $A$ has a pattern as multipotent in properties as that of the corresponding typedeterminant receptor in Type $\mathbf{A}$ of the typhoid bacillus, otherwise phage $\mathbf{A}$ would be able to attack all Vi-types of the organism to the same titre instead of being, as it is, highly specific for Type A. It is probable, therefore, that the specific receptor of phage $A$ fits only a restricted portion of the multipotent type-determinant receptor zone of Type $A$, and that the corresponding specific receptors in all the adapted Vi-typing preparations are modifications of it.

\section{Serological relationships and other properties of latent phages}

No serological relationship was found between any of the latent phages and Vi-phage II. The majority of the type-determining phages belong to one serological group (group 3) comprising two subgroups. The six phages in this group share a number of other properties, although they are responsible for the specificity of different Vi-types. Thus, they are mostly poor antigens, are heat-labile and form micro-plaques on solid media; they are all stable to treatment with $0 \cdot 16 \%$ toluene. Phages $d 6, f 2,29^{\prime}$ and $30^{\prime}$, which by the technique so far employed are serologically indistinguishable, are more closely related to each other than to phages $25^{\prime}$ and $26^{\prime}$ in that the former induce in Type A, and in other types, resistance patterns exhibiting striking similarities. This is illustrated in Table 9.

It can be seen from Table 9 that Type $A$ is fully susceptible to all the Vityping phages used in the test. Type A made lysogenic with phage $d 6$ is resistant to phage $A$ in a concentration 20 times that used for routine phage typing. There are residual sensitivities left to the adapted Vi-phages F 2, 29 and 30-a feature sometimes found in naturally occurring Type D6-but sensitivity to all the other Vi-typing phage preparations, which are not 
included in the table for the sake of brevity, has largely or completely disappeared. Sensitivity to Vi-phage $I$ is unchanged but the organism has become resistant to Vi-phage IV and to the polyvalent anti-O phage. The phagesensitivity spectrum of Type A, after treatment with phages $f 2$ and $29^{\prime}$, is identical and the organism, in fact, has become Type 29. On the other hand, Type A made lysogenic with phage $30^{\prime}$ becomes Type 29 with the difference that this artificially prepared type is fully sensitive to Vi-phage IV, whereas the natural Type 29 and those prepared artificially from Type A with phages f2 and $29^{\prime}$ are partially resistant to Vi-phage IV (see also Table 5).

\section{Table 9. Production of lysogenicity in Vi-phage Type $A$ with phages $d 6, f 2,29^{\prime}$ and $30^{\prime}$}

Reactions of resultant cultures to routine test dilutions of

\begin{tabular}{|c|c|c|c|c|c|c|c|c|}
\hline & \multicolumn{4}{|c|}{ Adapted Vi-typing phages } & & \multicolumn{2}{|c|}{$\begin{array}{l}\text { Unadapted } \\
\text { Vi-phages }\end{array}$} & \multirow{2}{*}{$\begin{array}{l}\text { Poly- } \\
\text { valent } \\
\text { anti-O } \\
\text { phage }\end{array}$} \\
\hline & $\mathbf{A}$ & D6 & F2 & 29 & $\mathbf{3 0}$ & I & IV & \\
\hline Cultures & $1500^{*}$ & 10000 & 5000 & 20000 & 10000 & 100 & 100 & 30000 \\
\hline Type A & $\mathbf{C L}$ & $\mathbf{C L}$ & $\mathrm{CL}$ & $\mathbf{C L}$ & $\mathbf{C L}$ & CL & $\mathbf{C L}$ & CL \\
\hline$\frac{A}{\text { Phage } d 6}$ & - & CL & $\mu$ & $\mu$ & $\mu$ & $\mathbf{C L}$ & $\mu$ & $\mu$ \\
\hline$\frac{\text { A }}{\text { Phage } f 2}$ & $\pm s$ & CL & CL & CL & CL & CL & $\mu$ & $\mu$ \\
\hline$\frac{\text { A }}{\text { Phage } 29^{\prime}}$ & $\pm s$ & $\mathbf{C L}$ & CL & CL & CL & $\mathbf{C L}$ & $\mu$ & $\mu$ \\
\hline$\frac{\text { A }}{\text { Phage } 30^{\prime}}$ & $\pm s$ & CL & CL & CL & CL & CL & CL & $\mu$ \\
\hline
\end{tabular}

Another difference between the four phages in subgroup $3 a$ is disclosed when Type $F 1$ is employed as the substrate strain in type-transformation experiments (see Table 5). Phage $f 2$ rapidly transforms Type F1 into F2, whereas phages $d 6,2^{\prime}$ and $30^{\prime}$ can only effect this transformation with considerable difficulty. It is thus evident that, although very closely similar in many respects, including their serological reactions, phages $d 6, f 2,2^{\prime}$ and $30^{\prime}$ are nevertheless distinct phages.

Serological group 1 (see Table 8) comprises only two phages, $b 3$ and 28', which appear to be serologically identical. Up to the present it has not been possible to show that these phages, though carried by two different Vi-types of Sal. typhi, have a type-determining function. As these phages have identical sensitivities to physical and chemical agents and identical lytic spectra they are, for the time being, indistinguishable from each other. 
Serological group 2 contains phages which have several interesting features. Phages $d 1$ and $d 4$ appear to be identical in all respects. They give complete cross-neutralization, are heat stable, have the same lytic spectrum and transform Type A into Type D1. Consequently, the difference between Types D 1 and $\mathrm{D} 4$ cannot be ascribed to phages $d 1$ and $d 4$, but is due to some other, as yet unidentified, factor. Phage $k$ has so far only been shown to make cultures resistant to the polyvalent anti-O phage, although it differs from the latter in being thermolabile. The serological relationship between phages $d 1$ and $d 4$, on the one hand, and phage $k$ and anti-O phage no. 2 on the other, has not been found to be associated with other similarities between these phages.

A study of Table 5 will show that the identity of a Vi-type of Sal. typhi prepared artificially in the laboratory depends on two factors: the phage employed to carry out the change and the original Vi-phage type of the culture subjected to the treatment. When the manipulation is carried out with Type $\mathbf{A}$ as the substrate organism, it is not always possible to reproduce the lysogenic type from which a latent phage has been isolated. To accomplish this it is often necessary to choose one of the known specific Vi-phage types as substrate. A clue to the choice of organism of the correct type is often provided by the knowledge of the Vi-phage reactions of the original type strain and of the lytic reactions of the adapted Vi-typing phage prepared for it from Vi-phage II, bearing in mind the fact that the type-determining phages can diminish but not increase the spectrum of sensitivity of a strain to the Vi-typing phages. For example, Type 30 when first examined gave a few plaques with the routine test dilution of phage $C$, and Type $C$ was found to be fully sensitive to the newly adapted Vi-typing phage 30 which was prepared by growing phage $\mathbf{A}$ on Type 30 . When the latent phage $30^{\prime}$ was isolated it was found to lyse Type $C$ and to convert it into Type 30. Knowing that phage $f 2$ converted Type A into Type 29 , which is sensitive to Vi-phage 30, and that the latent phage $30^{\prime}$ effected a similar transformation of Type $\mathbf{A}$, it was predicted that if Type $C$ were sensitive to phage $f 2$, the latter would convert it into Type 30; this was found to be the case.

Since the type-determining phages can only diminish the range of phage sensitivity of a strain it would not be expected that Type E 1 treated with phage $d 1$ would become Type D1, as Craigie (1946) reported in his early experiments. This would necessitate not only the blocking of reactivity of the strain to Vi-phages $\mathbf{E} 1$ and E2, but also the opening up of the previously blocked reactivity to Vi-phages of the $\mathbf{D}$ group. For this reason we predicted that such treatment would result in the production of a strain untypable with the existing adapted Vi-phage preparations. This expectation proved to be correct.

\section{Theory and practice of bacteriophage-typing}

As has been pointed out previously (Felix \& Anderson, 1951a), the fact that Vi-types can be prepared artificially does not diminish the epidemiological reliability of the Vi phage-typing method. The constancy of types characteristic of each country, and of different areas of the same country, and the 
fact that known carriers have been shown to excrete the same type of Sal. typhi over a period of years, furnish sufficient proof that the chances of the typhoid bacillus encountering type-changing phages in nature are remote.

It is necessary here to discuss the suggestion made recently by Scholtens (1950), Boyd (1950) and Boyd et al. (1951) of typing bacteria by identifying the phages they carry. Although the theoretical aspects of lysogenicity are, of course, of fundamental importance, a consideration of the properties of the latent phages of the typhoid bacillus described in the present paper indicates that the suggested method of typing would be unsuitable for application to Sal. typhi. The main reasons for this unsuitability are:

(1) Different Vi-phage types may carry the same phage, or phages so alike that their differentiation is extremely difficult. Examples are Types D6, F2, 29 and 30, all of which carry thermolabile micro-plaque phages that are serologically indistinguishable, and whose lytic spectra are closely similar, but frequently unidentifiable, because of the inconstancy of the lysis they produce on solid media. Strains of Sal. typhi belonging to these four Vi-phage types would almost certainly be classified as being identical by the suggested method of identifying the latent phages. In contrast, the strains are easily distinguishable from each other by the adapted Vi-typing phages.

(2) Strains from which phages have not been isolated would be grouped together under the designation of 'phage undetermined'. On this basis no less than eighteen of the thirty recognized Vi-phage types of Sal.typhi would have to be abandoned for the time being.

(3) The isolation and identification of the latent phages of the typhoid bacillus are often extremely difficult and time-consuming manipulations. On the other hand, the routine Vi-phage typing technique is easy and will usually give a clear-cut result in $7 \mathrm{hr}$. with Sal. typhi.

These points make it evident that the typing of strains of Sal. typhi by a procedure analogous to that suggested by Boyd (1950) and Boyd et al. (1951) for Sal. typhimurium could not be compared in simplicity, speed and reliability with the Vi-phage typing method in current international use. A recent publication by Boyd (1952) shows full recognition of this fact.

An example of the errors that may arise as the result of the use of latent phage characterization for the identification of strains of Sal. typhi is provided by the observations of Henderson \& Ferguson (1949). These workers investigated two Vi types, DI and D4HP 56, and decided that they represented only a single type. They based this conclusion on two facts: first, that both types were being excreted by the same carrier, and secondly, that all the cultures, when examined by Craigie, yielded the same latent phage (see Henderson \& Ferguson (1949)). This phage is now designated phage $d 1$ and is identical with $d 4$, and strain D4HP56 is identical with Type D4 described by Felix (1943). Unpublished observations of our own have shown that cultures of Type D4, maintained for a long time in the laboratory, in rare instances throw off Type $D 1$ variants. This change is not accompanied by the loss of phage $d \mathbf{1}$ but is apparently more closely allied to the process, or processes, of 'degradation' in the non-lysogenic specific types that lead to the emergence 
of Vi-phage Type A. 'Degradation' is known to occur also in chronic carriers of long standing (Felix, unpublished observations).

Type $\mathbf{D} 4$ is, in our experience, a valid epidemiological type that is easily distinguishable from Type $D 1$ with the adapted Vi-typing phages, although the identification of phage $d 1$ which is carried by both types will not distinguish between $D_{1}$ and D4 strains. It was the identification of Type D4 and the story of a puzzling outbreak due to this type (Felix, 1943, Bradley, 1943) that helped to convince the most sceptical epidemiologists and laboratory workers of the value of the then new laboratory technique. There is no more justification for merging Types $\mathrm{D} 1$ and $\mathrm{D} 4$ on the grounds adduced by Henderson \& Ferguson (1949) than there is for merging many of the other recognized types with Type A because they may, on rare occasions, throw off degraded variants reacting as Type $A$.

It is evident from the experiments described in this paper that the study of Vi-phage susceptibility in Sal. typhi and its relationship to the latent phages carried by this organism is one of considerable complexity, with the added drawback that there are many technical difficulties still to be overcome.

We gratefully acknowledge the valuable technical assistance received from Mr F. J. Flynn and Mrs E. T. Kiewe.

\section{REFERENCES}

ADAms, M. H. (1950). Bacterial viruses. In Methods in Medical Research, 2. Chicago: Year Book Publishers.

Anderson, E. S. (1951). The significance of Vi-phage Types F 1 and F 2 of Salmonella typhi. J. Hyg., Camb. 49, 458.

Anderson, E. S. \& Felix, A. (1952). Variation in Vi-phage II of Salmonella typhi. Nature, Lond. 170, 492.

Anderson, E. S. \& Felrx, A. (1953). 'Degraded Vi strains' and variation in Viphage II of Salmonella typhi. J. gen. Microbiol. 8, 408.

Boyd, J. S. K. (1950). The symbiotic bacteriophages of Salmonella typhi-murium. J. Path. Bact. 62, 501.

BoyD, J. S. K. (1952). Bacteriophage-typing and epidemiological problems. Brit. med. J. ii, 679.

Boyd, J. S. K., Parker, M. T. \& MaIr, N. S. (1951). Symbiotic bacteriophage as a 'marker' in the identification of strains of Salmonella typhi-murium. J. Hyg., Camb. 49, 442.

Bradley, W. H. (1943). An endemiological study of Bact. typhosum Type D4. Brit. med. J. i, 438.

Craigie, J. (1940). Variation of Type II Vi phage. Proc. Third Int. Congr. Microbiol., New York, 1939, p. 296.

Craigre, J. (1942). The present status of phage typing of Bact. typhosum. Canad. publ. Hith J. 33, 41.

Craigre, J. (1946). The significance and applications of bacteriophage in bacteriological and virus research. Bact. Rev. 10, 73.

Crajgie, J. \& Feurx, A. (1947). Typing of typhoid bacilli with Vi bacteriophages. Lancet, i, 823.

Craigie, J. \& Yen, C. H. (1938). The demonstration of types of B. typhosus by means of preparations of Type II Vi phage. Canad. publ. Hlth J. 29, 448, 484.

Feurx, A. (1941). A new type of typhoid and paratyphoid vaccine. Brit. med. $J$. i, 391. 
Felrx, A. (1943). Experiences with typing of typhoid bacilli by means of Vi bacteriophage. Brit. med. J. i, 4335 .

Fentx, A. \& Anderson, E. S. (1951a). Bacteriophages carried by the Vi-phage types of Salmonella typhi. Nature, Lond. 167, 603.

Feltx, A. \& Anderson, E. S. (1951b). Bacteriophage, virulence and agglutination tests with a strain of Salmonella typhi of low virulence. J. Hyg., Camb. 49, 349.

Felix, A. \& Callow, B. R. (1943). Typing of paratyphoid-B bacilli by means of Vi bacteriophage. Brit. med. J. ii, 127.

Fenix, A. \& Callow, B. R. (1951). Paratyphoid-B Vi-phage typing. Lancet, ii, 10.

Gratia, A. (1936). Des relations numériques entre bactéries lysogènes et particules de bactériophage. Ann. Inst. Pasteur, 57, 652.

Hamon, Y. \& Nrcolce, P. (1951). Recherches sur les facteurs qui conditionnent l'appartenance des bacilles paratyphiques $B$ aux différents types bactériophagiques de Felix et Callow. II. Ann. Inst. Pasteur, 80, 496.

Henderson, N. D. \& Ferguson, W. W. (1949). Investigation of bacteriophage types D1 and D 4HP 56 of Salmonella typhi and their latent bacteriophages. Amer. J. Hyg. 50, 349.

Jude, A., Niconne, P. \& Ducrest, P. (1951). Sur la présence simultanée de deux types bactériophagiques (D1 and D6) dans une culture de Salmonella typhi. Ann. Inst. Pasteur, 81, 245.

Nicolle, P., Grabar, J. \& Gibert, P. (1946). Fréquence de la lysogénéité et moindre fréquence apparente de la lysosensibilité parmi les bacilles paratyphiques B. Ann. Inst. Pasteur, 72, 81.

Nicolle, P., Hamon, Y. \& Edunger, E. (1951). Recherches sur les facteurs qui conditionnent l'appartenance des bacilles paratyphiques $B$ aux différents types bactériophagiques de Felix et Callow. I. Ann. Inst. Pasteur, 80, 479.

Scholtens, R. TH. (1950). Lysogenicity as a tool for bacteriophage typing of Salmonella paratyphi B. Leeuwenhoek ned. Tijdschr. 16, 256.

Williams Smith, H. (1951 a). Some observations on lysogenic strains of Salmonella. J. gen. Microbiol. 5, 458.

Williams Smrth, H. (1951b). The typing of Salmonella thompson by means of bacteriophage. J. gen. Microbiol. 5, 472.

Wrulams Smith, H. (1951c). The typing of Salmonella dublin by means of bacteriophage. J. gen. Microbiol. 5, 919.

\section{EXPLANATION OF PLATE}

Comparison of plaque sizes of Vi-phage A with those of latent phages $25^{\prime}, \mathbf{2 6}^{\prime}$ and $f 2$. All phages titrated on Type $A$ and incubated for $7 \mathrm{hr}$. at $38.5^{\circ} \mathrm{C}$. The heavy outer circle marks the limit of the phage-inoculated area. The light inner circle is an artefact due to the method of deposition of the bacterial lawn.

Fig. 1. Vi-phage $A ; \times 5 \cdot 5$.

Fig. 2. Phage 25; $\times 5.5$.

Fig. 3. Phage $26^{\prime} ; \times 5 \cdot 5$.

Fig. 4. Phage $f 2 ; \times 5 \cdot 5$. (The area photographed contained forty plaques visible with a $\times 10$ hand-lens; only five of the plaques are discernible in Fig. 4.) 
Journal of General Microbiology, Vol. 9, No. 1
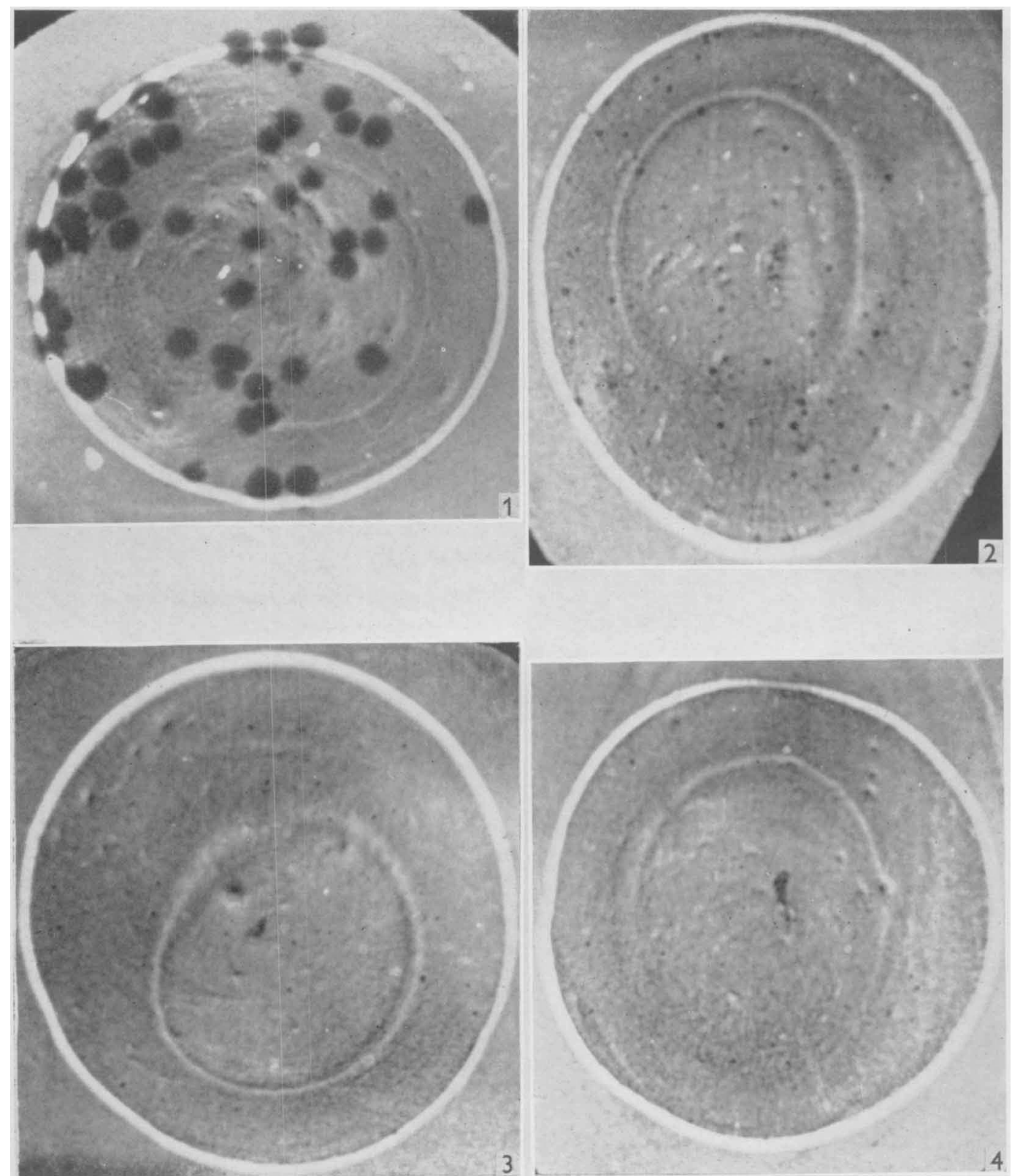

E. S. Anderson \& A. Felix-Vi type-inetermining phages of Salionella typhi. Plate 1 